Books, videos, cd-roms, dvds and any other relavant items submitted for a review in the bdj should be addressed to:

Mike Grace, Editor, British Dental Journal, 64 Wimpole Street WIG 8YS
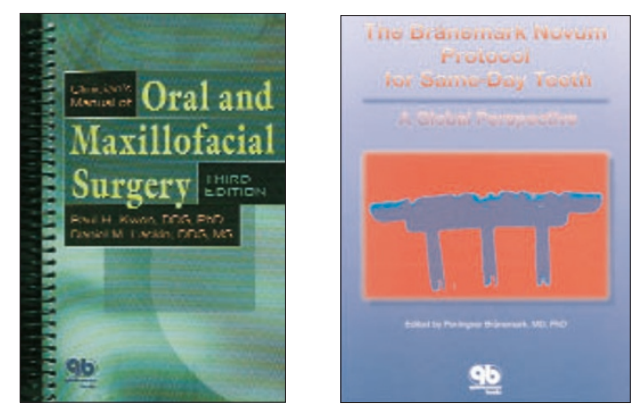

\section{Clinician's manual of oral and maxillofacial surgery}

\author{
P. H. Kwon \& D. M. Laskin 3rd ed \\ London: Quintessence Books, 2001 \\ price $€ 41$, pp432 ISBN 0-86715-396-2
}

This is the 3rd edition of this book, providing a concise series of 24 chapters, with contributions from 23 clinicians in addition to the editors.

The first half of the book looks clearly and concisely at aspects of patient management, investigations and medication in relation to the specialty of oral and maxillofacial surgery. It involves easily readable chapters on history and physical examination, appropriate laboratory tests and imaging techniques and a good overview of how to organise oneself within the hospital service particularly in relation to documentation. The book once again highlights the importance of keeping careful and correct records.

All the basic laboratory tests are covered as is ECG interpretation in its own chapter. Equally the chapters on fluids and electrolytes, surgical nutrition, blood and blood products provide an excellent overview of these topics. In the imaging chapter there is now additional information relating to ultra sound for deep vein thrombosis and a small addition on parenteral nutrition. The information on Haemophilia A and Haemophilia B and Von Willibrand's Disease has been expanded. The chapter on commonly used medications has been completely re-written and is like a mini formulatory, but should be used in consultation with the BNF. The chapter on managing medical emergencies has also been re-organised and improved, to make it easier to follow. In addition, the chapter on the management of the medically compromised patient has been expanded.

The chapter on post-operative problems provides a very good overview of techniques such as access to veins and arteries and maintaining a secure airway and finishes with a good overview of suturing procedures. The chapter on outpatient sedation, general anaesthesia and the management of anaesthetic emergencies once again is concise and factual on the different aspects of anaesthesia.

The manual then deals in more detail with specific aspects of oral and maxillofacial surgery. The chapter on the complications of dento-alveolar surgery looks at pre-operative medical assessment of the patient and how that may influence treatment. It includes local anaesthesia and possible complications and then moves on to discuss the variety of complications that may occur in relation to routine dentoalveolar surgery. Treatment planning for implant surgery follows in a new chapter providing a comprehensive overview of the techniques of implantology.

There is a comprehensive chapter on cysts and tumours, including classifications, radiological appearances and differential diagnoses. Infection in relation to oral and maxillofacial surgery is covered by considering clinical examination, the investigations that may be required including culture and sensitivity and drainage. There is a comprehensive review of antibiotics and the possible sequelae of infection, dealing in small detail with where infection may spread. The chapter on trauma is well laid out, and considers initial evaluation in relation to the general management of a patient with maxillofacial injuries. Soft tissue trauma is considered and information regarding different fractures of the facial skeleton is comprehensively covered. The chapters on temporo-mandibular joint and salivary gland disease are well presented providing aetiological factors, clinical presentation, methods of investigation, differential diagnosis and treatment of these conditions. The manual is completed by a general overview of dento-facial and cranio-facial abnormalities and facial aesthetic surgery.

This book remains a comprehensive userfriendly text which covers vast amounts of information relevant to the subject of oral and maxillofacial surgery. The emphasis remains on aids and investigations to enable appropriate differential diagnosis.
This provides a valuable text for clinicians at all levels, an excellent achievement for the editors.

\section{J. G. Cowpe [BR 5014]}

\section{The Brånemark Novum protocol for same day teeth: a global perspective}

Per-Ingvar Brånemark (ed)

London: Quintessence Books, 2001

price $£ 69$, pp168

ISBN 3-87652-634-5

This well illustrated book covers the provision of 'same day teeth' using the Brånemark Novum protocol. There is an excellent introduction to the concept of osseointegration that will be familiar to those working with dental implants. The innovative leap from the classic approach to a system where immediate loading is encouraged is covered and will fascinate the reader. This section contains an essay by the late Professor Richard Skalak covering the bioengineering principles of a one step implant system compared with a two step procedure for osseointegration and is a must-read for all those working in the field. The ground-breaking ideas of Professor Skalak provoke a re-evaluation of clinical practice and underpin what at first appears a controversial clinical technique that violates the prevailing hypothesis of osseointegration.

The major part of the book deals with case reports from various centres detailing their experiences with the Novum system. There is a lot of repetition in these reports, of basic technique, the reader will however be fascinated by the little gems of information relating to things that did not go to plan and how the various clinicians resolved these difficulties. This open sharing of information will be of immense value to those undertaking the clinical procedures described and it is to them whom this book is recommended. Ian M Brook [BR5013] 